\title{
Internal Heat Generation Effect on Thermal Boundary Layer with a Convective Surface Boundary Condition
}

\author{
P. O. Olanrewaju ${ }^{1, *}$, O.T. Arulogun², K. Adebimpe ${ }^{3}$ \\ ${ }^{1}$ Department of Mathematics, Covenant University, Canaanland, Km 10, Idiroko Road, Ota, Nigeria \\ ${ }^{2}$ Department of Computer Science and Engineering, Ladoke Akintola University of Technology, Ogbomoso, Nigeria \\ ${ }^{3}$ Department of Mathematical Sciences, Osun State University, Osogbo, Nigeria
}

\begin{abstract}
In this study, we examine the combined effects of internal heat generation and a convective boundary condition on the laminar boundary layer flow over a flat plate. It is assumed that lower surface of the plate is in contact with a hot fluid while a stream of cold fluid flows steadily over the upper surface with a heat source that decays exponentially. Using a similarity variable, the governing non-linear partial differential equations have been transformed into a set of coupled non-linear ordinary differential equations, which are solved numerically by applying shooting iteration technique together with fourth order Runge-Kutta integration scheme. The effects of Prandtl number, local Biot number, and the internal heat generation parameter on the velocity and temperature profiles are illustrated and interpreted in physical terms. A comparison with previously published results on special case of the problem shows excellent agreement
\end{abstract}

Keywords Blassius flow, Convective boundary condition, internal heat generation, Shooting method

\section{Introduction}

Laminar boundary layer about a flat-plate in a uniform stream of fluid continues to receive considerable attention because of its importance in many practical applications in a broad spectrum of engineering systems like geothermal reservoirs, cooling of nuclear reactors, thermal insulation, combustion chamber, rocket engine, etc. In a pioneering work, Blasius[1] presented a theoretical result for the boundary layer flow over a flat plate in a uniform stream and on a circular cylinder. Thereafter, several authors[2-6] have made significant advances in generalising his theoretical study to various situations of practical interest. Moreover, there are a number of physical circumstances where internal heat generation in an otherwise forced convective flow over a flat surface do occur. For instance, in the development of a metal waste form from spent nuclear fuel, phase change processes and thermal combustion processes, convection with internal heat generation plays an important role in the overall heat transfer process. Crepeau and Clarksean[7] considered the classical problem of natural convection from an isothermal vertical plate and added a heat generation term in the energy equation. They found that for a true similarity solution to exist, the internal heat generation must decay exponentially with the classical similarity variable.

In this present study, the combined effects of an exponen-

* Corresponding author:

oladapo_anu@yahoo.ie (P. O. Olanrewaju)

Published online at http://journal.sapub.org/ajfd

Copyright (C) 2012 Scientific \& Academic Publishing. All Rights Reserved -tially decaying internal heat generation and a convective boundary condition on the thermal boundary layer over a flat plate are investigated. Using a similarity approach, the transport equations are transformed to nonlinear ordinary differential equations and solved numerically using a shooting iteration technique together with fourth order Runge-Kutta integration scheme. The pertinent results are displayed graphically and discussed quantitatively.

\section{Mathematical Analysis}

We consider the steady flow of a stream of cold incompressible fluid at temperature $T_{\infty}$ over the upper surface of the flat plate with a uniform velocity $U_{\infty}$ while the lower surface of the plate is heated by convection from a hot fluid at temperature $T_{f}$ which provides a heat transfer coefficient $h_{f}$ as shown in Fig.1. The cold fluid in contact with the upper surface of the plate generates heat internally at the volumetric rate $\dot{q}$. The density variation in this fluid is taken into account using the Boussinesq approximation.

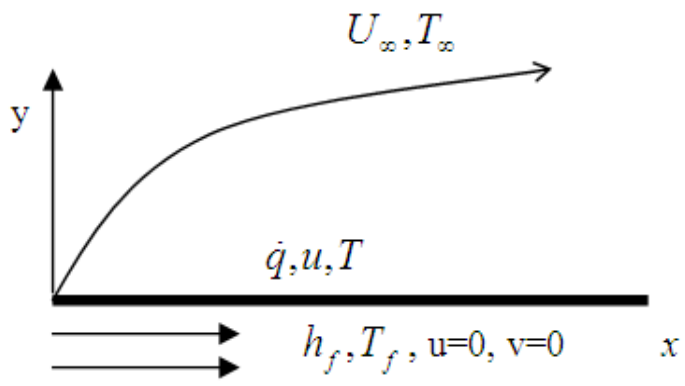

Figure 1. Flow configuration and coordinate system 
The continuity, momentum, and energy equations describing the flow can be written as

$$
\begin{gathered}
\frac{\partial u}{\partial x}+\frac{\partial v}{\partial y}=0 \\
u \frac{\partial u}{\partial x}+v \frac{\partial u}{\partial y}=v \frac{\partial^{2} u}{\partial y^{2}} \\
\rho c_{p}\left(u \frac{\partial T}{\partial x}+v \frac{\partial T}{\partial y}\right)=k \frac{\partial^{2} T}{\partial y^{2}}+\dot{q}
\end{gathered}
$$

where $u$ and $v$ are the $x$ (along the plate) and the $y$ (normal to the plate) components of the velocities, respectively, $T$ is the local temperature, $v$ is the kinematics viscosity of the fluid, $\rho$ is the fluid density, $c_{p}$ is the specific heat at constant pressure and $k$ is the thermal conductivity of the fluid. The velocity boundary conditions can be expressed as

$$
u(x, 0)=v(x, 0)=0, \quad u(x, \infty)=U_{\infty}
$$

The thermal boundary conditions at the plate lower surface and far into the cold fluid at the plate upper surface may be written as

$$
\begin{gathered}
-k \frac{\partial T}{\partial y}(x, 0)=h_{f}\left[T_{f}-T(x, 0)\right] \\
T(x, \infty)=T_{\infty}
\end{gathered}
$$

Introducing a similarity variable $\eta$ and a dimensionless stream function $f(\eta)$ and temperature $\theta(\eta)$ as

$$
\begin{gathered}
\eta=\frac{y}{x} \sqrt{\operatorname{Re}_{x}}, \quad u=U_{\infty} f^{\prime}, v=\frac{v}{2 x} \sqrt{\operatorname{Re}_{x}}\left(\eta f^{\prime}-f\right), \\
\theta=\frac{T-T_{\infty}}{T_{f}-T_{\infty}}, \lambda_{x}=\frac{\dot{q} x^{2} e^{\eta}}{k \operatorname{Re}_{x}\left(T_{f}-T_{\infty}\right)}
\end{gathered}
$$

where prime symbol denotes differentiation with respect to $\eta$ and $R e_{x}=U_{\infty} x / v$ is the local Reynolds number. The local internal heat generation parameter $\lambda_{x}$ is defined so that the internal heat generation $\dot{q}$ decays exponentially with the similarity variable $\eta$ as stipulated in[8]. This type of model can be used in mixtures where a radioactive material is surrounded by inert alloys and in the electromagnetic heating of materials[9]. After substituting Eq.(7) into Eqs. (1) - (6), we obtain the following locally similar equations:

$$
\begin{gathered}
f^{\prime \prime \prime}+\frac{1}{2} f f^{\prime \prime}=0 \\
\theta^{\prime \prime}+\frac{1}{2} \operatorname{Pr} f \theta^{\prime}+\lambda_{x} e^{-\eta}=0 \\
f(0)=f^{\prime}(0)=0 \quad \theta^{\prime}(0)=-B i_{x}[1-\theta(0)] \\
f^{\prime}(\infty)=1 \quad \theta(\infty)=0
\end{gathered}
$$

where

$$
B i_{x}=\frac{h_{f}}{k} \sqrt{\frac{v x}{U_{\infty}}}, \operatorname{Pr}=\frac{\rho c_{p} v}{k}
$$

The solutions generated whenever $B i_{x}$ and $\lambda_{x}$ are defined as in Eqs. (8)-(12) are the local similarity solutions. In order to have a true similarity solution the parameters $B i_{x}$ and $\lambda_{x}$ must be constants and not depend on $x$. This condition can be met if the heat transfer coefficient $h_{f}$ is proportional to $x^{-1 / 2}$ and the internal heat generation $\dot{q}$ is proportional to $x^{-1}$. In this case, we assume

$$
h_{f}=c x^{-\frac{1}{2}}, \dot{q}=l x^{-1}
$$

where $c$ and $l$ are constants but have the appropriate dimensions. Substituting Eq. (13) into Eqs. (7) and (12), we obtain

$$
B i=\frac{c}{k} \sqrt{\frac{v}{U_{\infty}}}, \lambda=\frac{l v e^{\eta}}{k U_{\infty}\left(T_{f}-T_{\infty}\right)}
$$

The Biot number $(B i)$ lumps together the effects of convection resistance of the hot fluid and the conduction resistance of the flat plate. The parameter $\lambda$ is a measure of the strength of the internal heat generation. We solved Eqs. (8)-(11) numerically using the fourth order Runge-Kutta scheme along with a shooting technique. The procedure was implemented in Maple[10] using a step size $\eta=0.001$. This step size was determined by running a step sensitivity test to ensure an accuracy of the results up to seven places of decimal. From the numerical results, the plate surface temperature, local skin friction coefficient and the local Nusselt number coefficient can easily be determined in terms of $\theta(0)$, $f^{\prime \prime}(0)$ and $-\theta^{\prime}(0)$, respectively.

\section{Results and Discussion}

For all values of the thermophysical parameters embedded in the system, the value of local skin friction coefficient represented by $f^{\prime \prime}(0)=0.332057$. Moreover, the local Nusselt number coefficient represented by $\theta^{\prime}(0)$ and the plate surface temperature $\theta(0)$ for different combination values of parameters are presented in Table 1 for the case of $\lambda_{x}=0$ (no internal heat generation) where a comparison with the results of Aziz[4] exhibits a perfect agreement up to five places of decimal. This degree of closeness vouches for the high accuracy of the present computational scheme. In Table 2, we highlight the effect of the internal heat generation on thermal boundary layer with respect to heat transfer characteristics of the flow. Note that we have tabulated the values of $\theta^{\prime}(0)$ and not $-\theta^{\prime}(0)$ as in Table 1, because except for one case, $\theta^{\prime}(0)$ is positive which means heat flows into the flat plate. For the conditions of weak plate heating ( $\left.B i_{x}=0.1\right)$, the data in the first and last two rows show that the local Nusselt number and the plate surface temperature increase rapidly as the local internal heat generation increases i.e. as $\lambda_{x}$ increases from 1 to 10 . As the plate heating becomes stronger i.e. as $B i_{x}$ increases from 0.1 to 10 , the local Nusselt number increases slightly with an increase in the back flow of heat into the plate while the plate upper surfaces temperature reduces slightly. With data in the first, fourth, and fifth rows of Table 2, one can see that as the $P r$ increases (from 0.72 Air to 7.1water) the surface temperature decreases curtailing the back heat flow into the plate. When $P r=7.10$, the normal heat flow direction (from the lower plate surface into the cold fluid) is restored. 
Table 1. Computations showing comparison with Aziz [2] results for $\operatorname{Pr}=0.72$

\begin{tabular}{|c|c|c|c|c|}
\hline$B i_{x}$ & $\begin{array}{c}\theta(0) \\
\text { Aziz [4] }\end{array}$ & $\begin{array}{c}-\theta^{\prime}(0) \\
\text { Aziz [4] }\end{array}$ & $\begin{array}{c}\theta(0) \\
\text { Present }\end{array}$ & $\begin{array}{c}-\theta^{\prime}(0) \\
\text { Present }\end{array}$ \\
\hline 0.05 & 0.1447 & 0.0428 & 0.1447 & 0.0428 \\
\hline 0.10 & 0.2528 & 0.0747 & 0.2528 & 0.0747 \\
\hline 0.20 & 0.4035 & 0.1193 & 0.4035 & 0.1193 \\
\hline 0.40 & 0.5750 & 0.1700 & 0.5750 & 0.1700 \\
\hline 0.60 & 0.6699 & 0.1981 & 0.6699 & 0.1981 \\
\hline 0.80 & 0.7302 & 0.2159 & 0.7302 & 0.2159 \\
\hline 1.00 & 0.7718 & 0.2282 & 0.7718 & 0.2282 \\
\hline 5.00 & 0.9441 & 0.2791 & 0.9442 & 0.2791 \\
\hline 10.00 & 0.9713 & 0.2871 & 0.9713 & 0.2871 \\
\hline 20.00 & 0.9854 & 0.2913 & 0.9854 & 0.2913 \\
\hline
\end{tabular}

Table 2. Computation showing $f^{\prime \prime}(0), \theta^{\prime}(0)$ and $\theta(0)$ for different parameter values

\begin{tabular}{|c|c|c|c|c|}
\hline $\mathrm{Bi}_{\mathrm{x}}$ & $\operatorname{Pr}$ & $\lambda_{x}$ & $\theta^{\prime}(0)$ & $\theta(0)$ \\
\hline 0.1 & 0.72 & 1 & 0.1154879 & 2.15487958 \\
\hline 1.0 & 0.72 & 1 & 0.3526541 & 1.35265410 \\
\hline 10 & 0.72 & 1 & 0.4437910 & 1.04437910 \\
\hline 0.1 & 3.00 & 1 & 0.0272290 & 1.27229008 \\
\hline 0.1 & 7.10 & 1 & -0.0101008 & 0.89899201 \\
\hline 0.1 & 0.72 & 5 & 0.8763365 & 9.76336572 \\
\hline 0.1 & 0.72 & 10 & 1.8273973 & 19.273973 \\
\hline
\end{tabular}

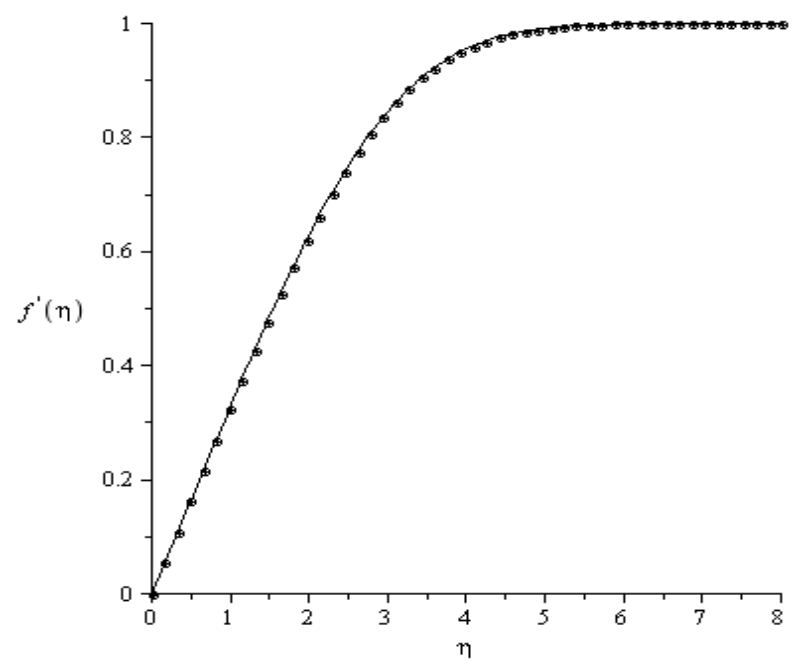

Figure 2. Velocity distribution

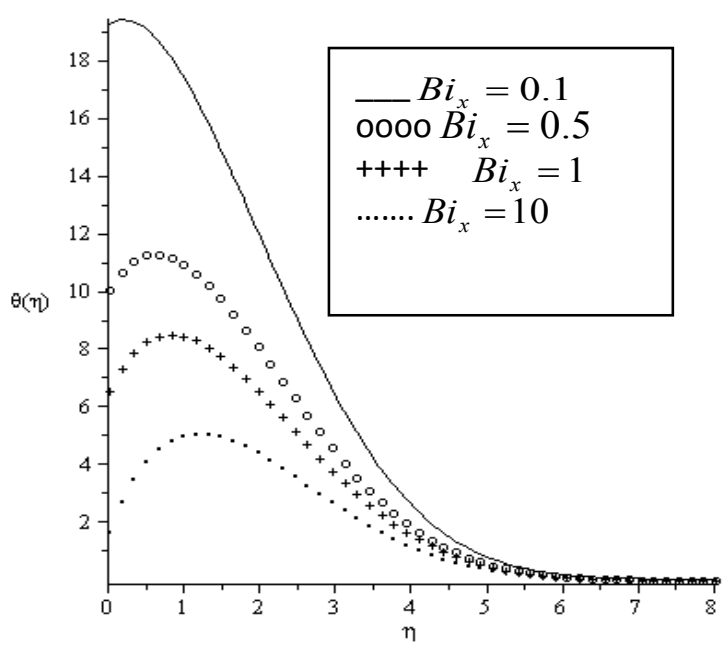

Figure 3. Temperature distribution for $\operatorname{Pr}=0.72, \lambda_{x}=10$

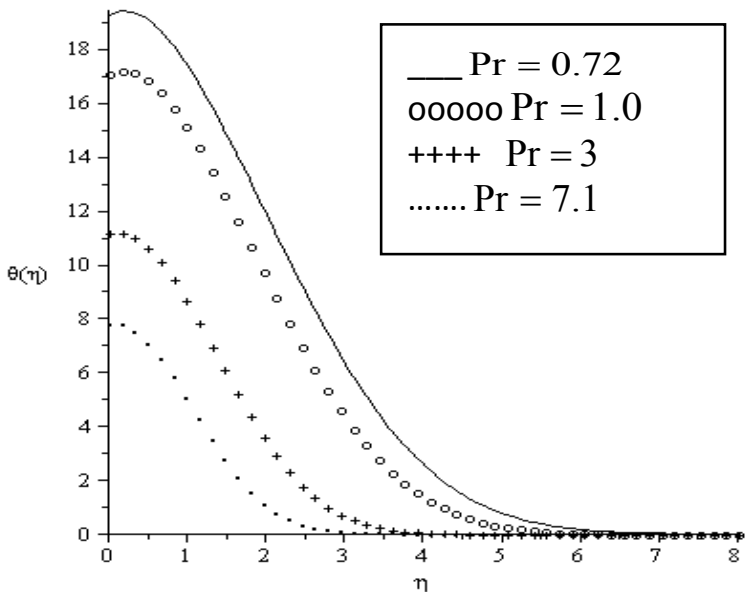

Figure 4. Temperature distribution for $B i_{x}=0.1, \lambda_{x}=10$

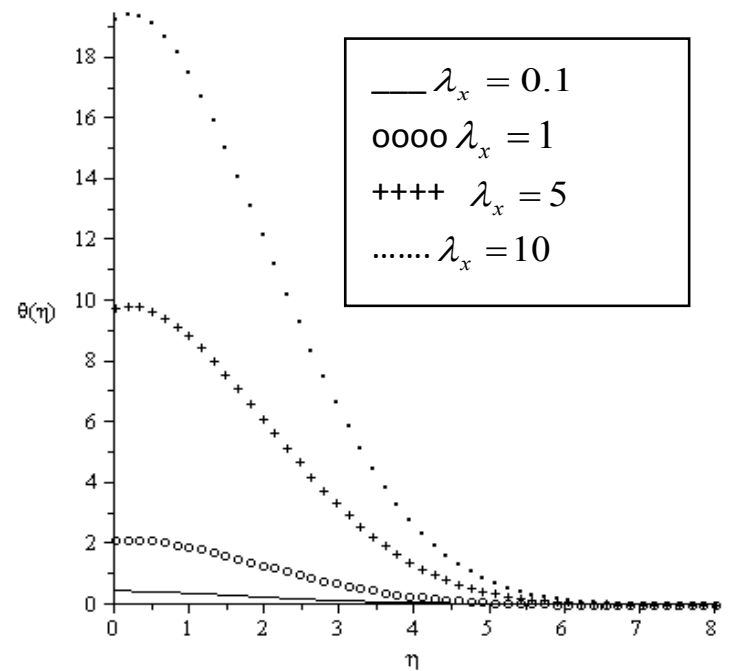

Figure 5. Temperature distribution for $P r=0.72, G r_{x}=B i_{x}=0.1$

Fig. (2) shows the sample of velocity profile. The fluid velocity is zero at the plate surface, increases rapidly to and attains its free stream velocity values far away from the plate satisfying the boundary condition. Figures (3)-(5) illustrated the effects of various parameters on the temperature profiles. Because of strong internal heat generation $\left(\lambda_{x}=10\right)$, Fig.3 shows that the plate surface temperatures exceed the temperature of the fluid on the lower surface of the plate and the direction of heat flow is reversed as noted in the earlier discussion. The peak temperature occurs in the thermal boundary in a region close to the plate. Although the temperature reduces as the local Biot number increases but the back heat flow persists. The effect of Prandtl number on the temperature distribution is depicted in Fig.4. As the Prandtl number increases, the thermal boundary layer thickness decreases, leaving less energy for the back heat flow Fig.5 reveals that only for weak internal heat generation i.e. $\lambda_{x}=$ 0.1 , the plate surface temperature is less 1 and heat is able to flow from the lower surface of plate into the fluid on the upper face of the plate. For all other values of $\lambda_{x}$, the heat flows back into the plate. It must be kept in mind that for Fig.5, the convection on the lower surface of the plate is rather weak $\left(B i_{x}=0.1\right)$ and consequently unable to push heat 
through the plate to the fluid on the upper face of the plate except when the internal heat generation is weak, e.g. $\lambda_{x}=$ 0.1 .

\section{Conclusions}

The effects of exponentially decaying internal heat generation on boundary layer flow over the upper surface of a flat plate with a convective boundary condition on its lower surface is investigated numerically. A local similarity analysis identified three governing dimensionless parameters: Biot number, internal heat generation parameter, and the Prandtl number. Our results reveal that the thermal boundary layer thickness decreases with an increase in the local Prandtl number and the local Biot number. An increase in the internal heat generation prevents the rapid flow of heat from the lower surface to the upper surface of the plate.

\section{Nomenclature}

$B i_{x}=$ local Biot number for hot fluid

$c_{p}=$ cold fluid specific heat

$f(\eta)=$ similarity function

$f^{\prime}(\eta)=$ dimensionless velocity

$h_{f}=$ convective heat transfer coefficient

$k=$ thermal conductivity of fluid on the upper surface of plate

$c, l=$ constants

$\operatorname{Pr}=$ Prandtl number

$\dot{q}=$ volumetric heat generation

$\operatorname{Re}_{x}=$ local Reynolds number

$T=$ cold fluid temperature

$T_{f}=$ hot fluid temperature

$T_{\infty}=$ cold fluid free stream temperature

$u, \mathrm{v}=$ velocity components

$U_{\infty}=$ free stream velocity

$x, \mathrm{y}=$ Cartesian coordinates Greek symbols

$\mathrm{P}=$ fluid density

$\eta=$ similarity variable $\lambda=$ Internal heat generation parameter $v=$ kinematic viscosity

\section{REFERENCES}

[1] Blasius, H., 1908, "Grenzschichten in Flussigkeiten mit kleiner reibung”, Z. Math. Phys. 56, pp 1-37.

[2] Incropera, F. P. and De Witt D.P., 1990, Introduction to heat transfer, John Wiley, New York, pp 496-497.

[3] Aziz, A., 2009, "A similarity solution for laminar thermal boundary layer over a flat plate with a convective surface boundary condition," Commum. Nonlinear Sci. Numer. Simulat. 14, pp 1064-1068.

[4] O. D. Makinde O. D. and Ogulu A. 2008, "The effect of thermal radiation on the heat and mass transfer flow of a variable viscosity fluid past a vertical porous plate permeated by a transverse magnetic field," Chemical Engineering Communications, Vol. 195, No. 12, 1575 -1584.

[5] Makinde O. D., 2009, "On MHD boundary-Layer flow and mass transfer past a vertical plate in a porous medium with constant heat flux,". Int. J. Num. Methods for Heat \& Fluid Flow, Vol. 19, Nos 3/4, 546-554.

[6] Makinde O. D. and Olanrewaju P. O. 2010, "Buoyancy effects on thermal boundary layer over a vertical plate with a convective surface boundary condition," Transactions ASME Journal of Fluids Engineering, Vol. 132, 044502(1-4).

[7] Crepeau J. C. and Clarksean R., 1997, "Similarity solutions of natural convection with internal heat generation," Transactions of ASME - Journal of Heat Transfer, Vol. 119, pp 184-185.

[8] Viskanta, R, 1988, "Heat transfer during melting and solidification of materials," Transactions of ASME - Journal of Heat Transfer, Vol. 110, pp 1205-1219.

[9] Sahin, A. Z., 1992, Transient heat conduction in semi-infinite solid with spatially decaying exponential heat generation, Int. Comm. Heat Mass Transfer, Vol. 19, pp 349-358.

[10] Heck, A., 2003, "Introduction to Maple", 3rd Edition, Springer-Verlag. 